Archived version from NCDOCKS Institutional Repository http://libres.uncg.edu/ir/asu/

\title{
Appalachľan
}

$\overline{\text { B O O N E, N O R T H C A R O L I N A }}$

\section{The Physical Sacrifice Of Thinking: Investigating The Relationship Between Thinking And Physical Activity In Everyday Life}

By: Todd McElroy, David L. Dickinson, Nathan Stroh, and Christopher A. Dickinson

\begin{abstract}
Physical activity level is an important contributor to overall human health and obesity. Research has shown that humans possess a number of traits that influence their physical activity level including social cognition. We examined whether the trait of "need for cognition" was associated with daily physical activity levels. We recruited individuals who were high or low in need for cognition and measured their physical activity level in 30-second epochs over a 1-week period. The overall findings showed that low-need-for-cognition individuals were more physically active, but this difference was most pronounced during the 5-day work week and lessened during the weekend.
\end{abstract}

McElroy, T., et al. (2015). "The physical sacrifice of thinking: Investigating the relationship between thinking and physical activity in everyday life." Journal of Health Psychology 21(8): 1750-1757. https://doi.org/10.1177/1359105314565827. Publisher version of record available at: http://journals.sagepub.com/doi/full/10.1177/1359105314565827 


\title{
The physical sacrifice of thinking: Investigating the relationship between thinking and physical activity in everyday life
}

\author{
Todd McElroy', David L Dickinson², Nathan \\ Stroh $^{2}$ and Christopher A Dickinson ${ }^{2}$
}

\begin{abstract}
Physical activity level is an important contributor to overall human health and obesity. Research has shown that humans possess a number of traits that influence their physical activity level including social cognition. We examined whether the trait of "need for cognition" was associated with daily physical activity levels. We recruited individuals who were high or low in need for cognition and measured their physical activity level in 30-second epochs over a I-week period. The overall findings showed that low-need-for-cognition individuals were more physically active, but this difference was most pronounced during the 5-day work week and lessened during the weekend.
\end{abstract}

\section{Keywords}

cognition, decision, obesity, physical activity, risk

It has been clear for some time that physical activity level is an important contributor to human health (e.g. Pate et al., 1995) and is especially influential in overall quality of life (Bize et al., 2007). The importance of physical activity has recently garnered a great deal of attention because of the pandemic rise in obesity (Wang et al., 2011) and negative health effects (Kohl et al., 2012) associated with low activity levels. Perhaps more striking is that many people believe they can overcome their lack of physical activity by taking a quick run through the gym. While this is likely helpful, its effects on overall human health are minimal compared to the impact of a person's overall daily activity level (Levine et al., 1999). Maintaining a proper balance of physical activity to promote good health and quality of life is a centerpiece of the American College of Sports
Medicine (ACSM) and has been shown to support mental well-being (Fox, 1999) and good mental health (Paluska and Schwenk, 2000). Aside from physical activity, another domain in which people vary is how much they like to think, and one of the most widely used measures of differences in thinking propensity is "need for cognition" (NFC). NFC is defined as a tendency to engage in and enjoy effortful cognitive endeavors (Cacioppo and Petty, 1982). NFC is an individual

\footnotetext{
'Florida Gulf Coast University, USA

${ }^{2}$ Appalachian State University, USA

Corresponding author:

Todd McElroy, Department of Psychology, Florida Gulf Coast University, I050 I FGCU Blvd, South Fort Myers, FL 33965, USA.

Email: toddmcelroyfgcu@gmail.com
} 
difference measure of thinking that allows researchers to study thinking without placing demands on cognitive resources. Furthermore, NFC is driven by intrinsic motivation and is relatively stable across a person's lifetime (Cacioppo et al., 1996). Researchers have used this measure for over 30 years to examine the relationship between enjoyment of effortful cognitive endeavors and other variables related to cognition (Cacioppo et al., 1996).

For example, research has shown that individuals high in NFC appear to perform better on memory tasks (Boehm, 1994; Cacioppo et al., 1983), are generally more positive toward cognitively difficult tasks (Cacioppo et al., 1996), spend more effort when making decisions (Verplanken et al., 1992) and can make better ones (Levin et al., 2000). Low-NFC individuals have been shown to rely more on peripheral information (Cacioppo and Petty, 1982) and contextual cues such as attractiveness or a person's mood (Cacioppo et al., 1996) when thinking and forming attitudes.

Overall, these types of studies depict a psychometric tool that reveals an important force behind human cognition and its effects on everyday life. In the current investigation, we explore how NFC may be associated with daily physical activity levels. Although previous research has not specifically examined such a connection, related research suggests that it may exist.

\section{Cognition and physical activity}

The relationship between cognition and physical activity is important for health concerns, but it also speaks to a more fundamental question of how cognition interacts with the physical body across the human lifespan. Research looking at children and adolescents has shown many cognitive variables that are related to physical activity, including preferences, intentions (Sallis et al., 2000), and self-efficacy (Strauss et al., 2001). There is also evidence that neuroanatomical and neurochemical differences are linked to more pervasive behavioral disorders such as attention deficit hyperactivity disorder
(ADHD) (Swanson et al., 1998) and anxiety (Fride and Weinstock, 1988). Similarly, research looking at older adults has also consistently shown a relationship between physical activity and cognitive decline (Laurin et al., 2001; Weuve et al., 2004). Thus, the relationship between cognition and physical activity is an important question for the human experience, and the interaction likely extends across the lifespan (e.g. Heyn et al., 2004; Kramer and Erickson, 2007).

Research has also revealed a number of individual difference variables that appear to be associated with physical activity levels. For example, intention to perform a physical activity (e.g. Petty et al., 2013) is associated with physical behavior. Individual difference variables such as approach/avoidance motivation (Hevey and Dolan, 2014), "Health Types" (McGinty et al., 2012), sensation seeking in adolescents (Sallis et al., 2000) and some components of the Big Five personality traits (Rhodes and Smith, 2006) influence the likelihood of a person performing certain types of physical activities. Second, there is strong evidence that cognition is related to physical activity in daily life. This idea is highlighted in a large-scale study by Godin et al., (2010). In this study, Godin et al. tested the effects of several variables on physical activity. These variables were grouped by either social structure, which represents a person's hierarchical status, or social cognition, which involves the processing of social information. The findings revealed that social structure had only a small effect on physical activity, whereas social cognition was determined to be the key factor in predicting physical activity level.

Thus, existing research supports the view that cognition and physical activity level are associated, yet an important question is whether individual preference toward cognitive endeavors is associated with more or less physical activity. While research has not directly examined this question, our search identified studies that provide differing clues for how this relationship may unfold, and they provide a basis for our investigation. 
First, it is possible that high- and low-NFC individuals may be engaging in different strategies that ultimately affect their physical behavior. Specifically, it could be that an associativedissociative attentional strategy (Masters and Ogles, 1998) dichotomy may be at play such that low-NFC individuals engaged in more physical activity because they are better able to dissociate themselves from cues related to physical exertion (e.g. Stanley et al., 2007). In other words, this dissociation would make physical activity seem easier to these individuals. Some support for this can be found in a study by Watt and Blanchard (1994). In this study, low-NFC individuals demonstrated a greater propensity toward boredom and more strongly experienced its associated negative effect. High-NFC individuals appear to avoid this because of their ability to provide their own mental stimulation. Thus, high-NFC individuals seem more content to "entertain themselves" mentally, whereas low-NFC individuals quickly experience boredom and experience it more negatively.

In another study that involved a limited behavioral task, participants were charged with observing directionality of dots on a computer monitor. The findings showed that low-NFC individuals performed better individually than collectively, and they tended to outperform high-NFC persons during individual performance (Smith et al., 2001). This suggests that low-NFC individuals may "loaf" more in groups but may be more active at the individual level.

While these studies seem to suggest that low NFC will be associated with more physical activity, another set of findings seems to suggest an opposite relationship. For example, a study by Hess et al. (2011) looked at longitudinal effects of cognitive motivation across a wide age range. They combined Personal Need for Structure (PNS) with NFC scores to create a composite measure of cognitive motivation. Their results showed that this cognitive motivation measure was positively associated with social activities and interactions. Thus, this study would seem to suggest that high-NFC individuals may be more physically active in their daily lives. This finding is consistent with research showing that high-NFC individuals have a stronger tendency to seek out information (Verplanken et al., 1992), and they appear more motivated (Cacioppo et al., 1983).

\section{Summary and predictions}

Our review of the literature reveals good evidence that individual differences as well as cognition appear to be associated with physical activity. However, the direction of this relationship is not clear. One set of findings seems to suggest a tradeoff of sorts between cognitive and physical activity. Because high-NFC individuals are more content and eager to be involved in cognitive activities, the natural outcome is that they may be less physically active. On the other hand, another set of findings seems to suggest that high NFC may reflect an overall increase in motivation level that could lead to greater exploration of the environment and social activities. Thus, because our assessment of findings in the literature appears to present a contradictory picture, we designed the current investigation as a way to test this relationship and determine whether a person's level of cognitive activity is associated with more or less physical activity.

\section{Method}

\section{Participants and design}

The participants in this study were 30 high- and 30 low-NFC individuals; 45 of the participants were female. ${ }^{1}$ The conditions were roughly equal in regard to gender; 20 females were in the highNFC condition and 25 in the low-NFC condition. All participants were undergraduate students at Appalachian State University. The experiment utilized a one-way factorial design. The independent variable in this study was NFC level (high or low), and the dependent variable was the participant's activity levels across 1 week.

\section{Procedure}

The primary screening procedure was conducted through an online survey using the SONA 
software system. This selection method was necessary for several reasons: the relative scarcity of low-NFC individuals in our sample population, the week-long sampling period, and the monetary expense associated with compensating each participant. In this initial session, participants were informed about the nature of the study, including the potential for participation in the second stage. They were then asked to complete the NFC scale (Cacioppo et al., 1984). After completing the NFC scale, participants were awarded credit for their participation in this initial screening stage.

Next, we established criteria for discerning individuals who were high or low in NFC. Relying on our initial NFC screening as a sample population, we used the upper and lower 10 percent of our distribution as the criteria for determining our maximum and minimum scores for categorizing high and low NFC. Participants who were eligible to take part in the study were contacted via recruitment email; those who responded affirmatively were scheduled for an initial lab meeting. As a result of this recruitment classification method, high-NFC participants had NFC scores in the range of (4-62), whereas the range for low-NFC participants was $(-11$ to -39$)$.

The observation weeks were arranged ahead of time so that they occurred during the semester and did not include holidays. Participants were contacted via email to set up the initial lab meeting. Prior to the initial lab meeting, actigraphy devices (described below) were configured and assigned to each participant. During the initial lab meeting, participants were informed about the study and how to wear the device. Participants were instructed to carry out their typical daily routines. They were then assigned a follow-up lab meeting time. The follow-up lab meeting took place approximately 1 week later; scheduling was based on participants' availability. We chose a 1-week observational period because prior research has shown this to be the desirable time period for assessing variability in activity patterns (Matthews et al., 2002).

In the final lab meeting, participants returned their actigraphy devices, and they were compensated US\$10.00 each for participation and return of the device. They were given an overview of the nature of the study and also offered an output of their daily activity level data, which we promised to send them after the study concluded, and the data were scored. At the end of the final lab meeting, participants were asked several sets of questions unrelated to this study. Data from the actigraphy devices were downloaded using the manufacturer's software. Time periods when the device was removed, which were rare, were cleared from the dataset to avoid miscounting them as periods of zero activity. Sleep episodes were also removed from the data. The 1 -week period of measurement yielded $\sim 20,000$ activity measurements per participant.

\section{Materials}

To assess participants' level of NFC, we used the NFC scale (Cacioppo et al., 1984). This scale consists of 18 items; half have positive orientations and half contain negative orientations. Participants indicated how much they agreed or disagreed with each item on a 9-point scale ranging from very strong disagreement $(-4)$ to very strong agreement $(+4)$. Total scores on this scale range from 72 to -72 .

To measure participants' activity levels, we used an actigraphy device. The device is an accelerometer worn on the non-dominant wrist as a common means for measuring gross motor activity (Ancoli-Israel et al., 2003). This device resembles a common wrist watch and can be conveniently worn by participants. Measurement is made by internal accelerometers with sensitivity of $.05 \mathrm{~g}$-force. This sensitivity generates "activity counts" of varying strength and frequency during each time epoch used for data collection. We set the data sampling for the device to occur at epoch lengths of 30 seconds. The device is impact resistant, waterproof to $1-\mathrm{m}$ depth for 30 minutes, and can be worn 24 hours a day with few exceptions.

\section{Results}

After completion of the study, daily activity counts were obtained by averaging the 30 -second 


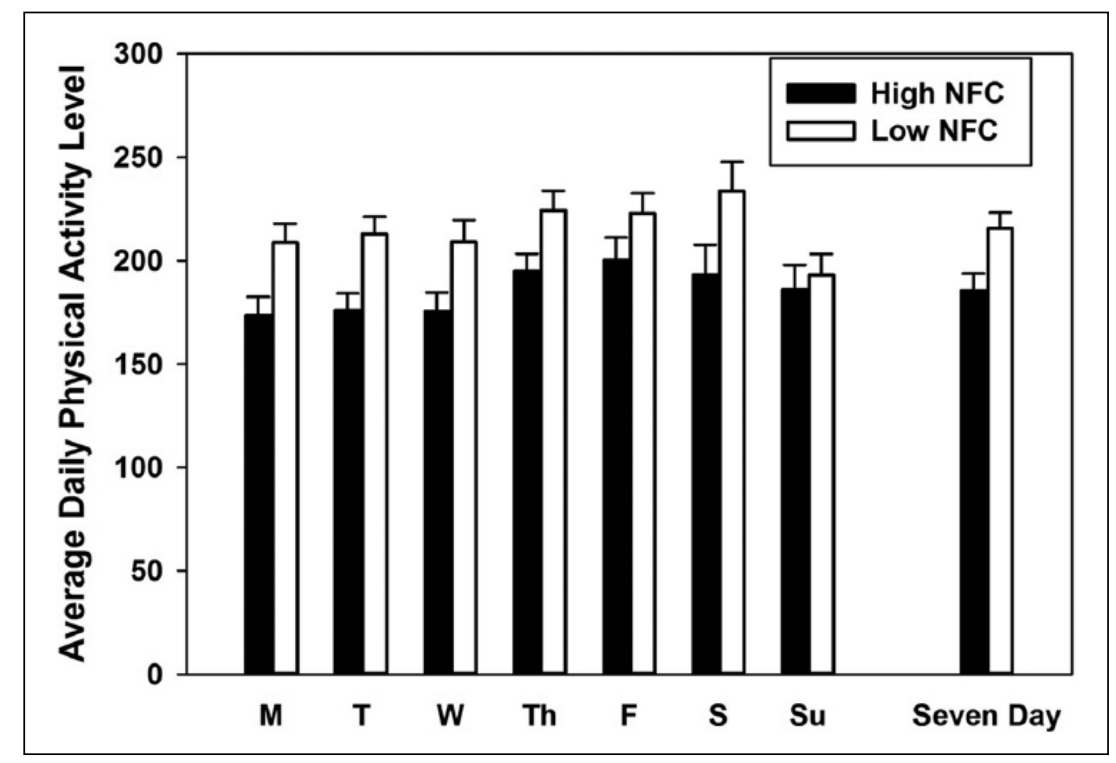

Figure I. Average daily physical activity level for high-NFC individuals and low-NFC individuals as measured in 30-second epochs and based on $.05 \mathrm{~g}$-force sensitivity. These data include the average daily physical activity level for each group across the I-week period. Error bars indicate the standard error of the mean.

epoch readings across all waking hours for each individual participant. There was a malfunction with the actigraphy for 1 day of a low-NFC participant's data, and so that day was not obtainable and excluded from the analysis. With this exception, the daily activity counts for each participant were combined within each of the 7 days; subsequent analyses relied on the entire daily activity counts within each individual day.

Because the one-way analysis of variance (ANOVA) $F$-test and $t$-test produce statistically identical outcomes, we relied on the ANOVA because the analysis yields more variance information. First, to test whether a person's level of NFC was associated with his or her physical activity levels, we performed an ANOVA with NFC level as our independent variable and overall daily activity level as our dependent variable. The results from this analysis revealed that thinking does seem to be associated with less physical activity. As shown in Figure 1, the difference between high- and low-NFC individuals in overall weekly physical activity level was highly significant $\left(F(1,58)=7.4, p<.009, \eta^{2}=.113\right)$ such that high-NFC individuals were far less active overall than low-NFC participants.

Prior research directed toward measuring daily differences in physical activity levels has shown that weekday activity levels (MondayFriday) differ substantially from weekend levels (Matthews et al., 2002). To test whether this weekend effect might be present in this study, we first performed an analysis of the weekday activity levels comparing high- and low-NFC individuals for Monday-Friday activity levels (see Figure 1), and, as suspected, they differed greatly across the 5-day typical work week $\left(F(1,58)=9.94, p<.003, \eta^{2}=.146\right)$. Next, we tested whether this effect remained for the weekend days. Collapsing across the weekend days, we see that activity levels for high- and low-NFC individuals did not differ significantly $\left(F(1,58)=2.53, p<.117, \eta^{2}=.042\right)$ on the weekend. The results revealed that this lack of a statistical difference in activity levels is true for Saturday data $\left(F(1,58)=2.4, p<.127, \eta^{2}=.04\right)$ and even more so for Sunday data $(F(1,58)=.21$, $\left.p>.65, \eta^{2}=.004\right) .^{2}$ 


\section{Discussion}

In this study, we tested whether people who prefer to think more will be less physically active in their daily lives than people who do not prefer to think. Our findings build upon prior research (e.g. Godin et al., 2010) and provide support for our hypothesis by revealing robust physical activity level differences during the 5-day work week and attenuated differences over the weekend. Furthermore, it is important to note that these differences were found using a robust measure of physical activity over a 1 -week period. This type of objective measure has been called for to help validate other types of self-report measures (see Godin et al., 2010). However, the sampling method used in our study created potential limitations that should be noted.

First, it is important to note that part of the "weekend effect" in our study may be due to our sample population, which consisted of college students. Although college students are a standard participant pool in the vast majority of experimental psychology studies, their behavior and habits may be more indicative of young adult behavior than adult behavior in general. It is reasonable to assume that this "weekend effect" may change as people progress through different life stages, which is a question that future researchers may want to consider. A similar limitation with our methodology was that the participants were all involved in coursework, a time in their lives that should revolve around cognitively focused events. While this was true for both high- and low-NFC participants, it may limit the external validity of this study to cognitively oriented life situations. In conclusion, it seems noteworthy to point out that if this association between physical activity and cognition leads to health issues such as obesity, it may be prudent for more thoughtful individuals to consider lifestyle changes as countermeasures to the negative health outcomes associated with their lower activity levels. For example, research has shown that simply being active in mundane behaviors such as moving about, fidgeting, or even walking to the bathroom increases non-exercise activity thermogenesis (NEAT). These types of activities have been shown to expend excess energy the body has taken in, which will help avoid fat storage and promote leanness (Levine et al., 1999). An example of a more dramatic countermeasure would be to replace one's workstation with a walking treadmill desk. These have been shown to increase energy expenditure of $100 \mathrm{kcal} /$ hour in the neighborhood, which can result in substantial benefits (Levine and Miller, 2007). Ultimately, an important factor that may help more thoughtful individuals combat their lower average activity levels is awareness. Awareness of their tendency to be less active, coupled with an awareness of the cost associated with inactivity, more thoughtful individuals may then choose to become more active throughout the day.

\section{Acknowledgements}

Special thanks to Sarah Pollard for her devoted assistance with the early data gathering in the project. The data set of daily averages as well as the entire set of daily activity measurements is available from the first author.

\section{Declaration of conflicting interests}

The author(s) declared no potential conflicts of interest with respect to the research, authorship, and/or publication of this article.

\section{Funding}

The author(s) disclosed receipt of the following financial support for the research, authorship, and/or publication of this article: Partial support for this research was provided by the National Science Foundation (NSF Grant number: 1229067) and the Division of Research and Sponsored Programs, Appalachian State University. Some subject payments were funded by a grant from the Office of Student Research, Appalachian State University.

\section{Notes}

1. The study was approved by the University's Institutional Review Board (IRB No. 11-0067), which is governed by the Office of Research Protections. Written consent was obtained from all participants. 
2. We performed an additional analysis by collapsing weekdays and weekends into two separate variables. We then performed a repeated measures analysis with these two new variables as a within factor and NFC level as a between factor. This approach to treat our data as a mixed design yielded a marginally significant main effect for NFC; $F(1,58)=3.59, p<.07$, a non-significant main effect for the weekday/weekend variable; $F<1$ and a non-significant interaction $F<1$.

\section{References}

Ancoli-Israel S, Cole R, Alessi C, et al. (2003) The role of actigraphy in the study of sleep and circadian rhythms. American Academy of Sleep Medicine Review Paper. Sleep 26: 342-392.

Bize R, Johnson JA and Plotnikoff RC (2007) Physical activity level and health related quality of life in the general adult population: A systematic review. Preventive Medicine 45: 401-415.

Boehm LE (1994) The validity effect: A search for mediating variables. Personality and Social Psychology Bulletin 20: 285-293.

Cacioppo JT and Petty RE (1982) The need for cognition. Journal of Personality and Social Psychology 42(1): 116-131.

Cacioppo JT, Petty RE and Kao CF (1984) The efficient assessment of need for cognition. Journal of Personality Assessment 48(3): 306-307.

Cacioppo JT, Petty RE and Morris KJ (1983) Effects of need for cognition on message evaluation, recall, and persuasion. Journal of Personality and Social Psychology 45: 805-818.

Cacioppo JT, Petty RE, Feinstein JA, et al. (1996) Dispositional differences in cognitive motivation: The life and times of individuals varying in NFC. Psychological Bulletin 119(2): 197-253.

Fox KR (1999) The influence of physical activity on mental well-being. Public Health Nutrition 2: 411-418.

Fride E and Weinstock M (1988). Prenatal stress increases anxiety related behavior and alters cerebral lateralization of dopamine activity. Life Science 42: 1059-1065.

Godin G, Sheeran P, Conner M, et al. (2010) Social structure, social cognition, and physical activity: A test of four models. British Journal of Health Psychology 15: 79-95.

Hess TM, Emery L and Neupert SD (2011) Longitudinal relationships between resources, motivation, and functioning. The Journals of Gerontology, Series B: Psychological Sciences and Social Sciences 67: 299-308.

Hevey D and Dolan M (2014) Approach/avoidance motivation, message framing and skin cancer prevention: A test of the congruency hypothesis. Journal of Health Psychology 19: 1003-1012.

Heyn P, Abreu BC and Ottenbacher KJ (2004) The effects of exercise training on elderly persons with cognitive impairment and dementia: A meta-analysis. Archives of Physical Medicine and Rehabilitation 85: 1694-1704.

Kohl HW, Craig CL, Lambert EV, et al. (2012) The pandemic of physical inactivity: Global action for public health. The Lancet 380: 294-305.

Kramer AF and Erickson KI (2007) Capitalizing on cortical plasticity: Influence of physical activity on cognition and brain function. Trends in Cognitive Sciences 11: 342-348.

Laurin D, Verreault R, Lindsay J, et al. (2001) Physical activity and risk of cognitive impairment and dementia in elderly persons. Archives of Neurology 58: 498-504.

Levin IP, Huneke ME and Jasper JD (2000) Information processing at successive stages of decision making: Need for cognition and inclusion-exclusion effects. Organizational Behavior and Human Decision Processes 82: 171-193.

Levine JA, Eberhardt NL and Jensen MD (1999) Role of nonexercise activity thermogenesis in resistance to fat gain in humans. Science 283: 212-214.

Levine JA and Miller JM (2007) The energy expenditure of using a "walk-and-work" desk for office workers with obesity. British Journal of Sports Medicine 41: 558-561.

McGinty H, Dark-Freudeman A and West RL (2012) Health hopes and fears for the future in relation to health behavior and current health status. Journal of Health Psychology 18: 1509-1518.

Masters KS and Ogles BM (1998) Associative and dissociative cognitive strategies in exercise and running: 20 years later, what do we know? Sport Psychologist 12: 253-270.

Matthews CE, Ainsworth BE, Thompson RW, et al. (2002) Sources of variance in daily physical activity levels as measured by an accelerometer. Medicine and Science in Sports and Exercise 34: 1376-1381.

Paluska SA and Schwenk TL (2000) Physical activity and mental health: Current concepts. Sports Medicine 29: 167-180. 
Pate RR, Pratt M, Blair SN, et al. (1995) Physical activity and public health: A recommendation from the Centers for Disease Control and Prevention and the American College of Sports Medicine. JAMA: The Journal of the American Medical Association 273: 402-407.

Petty KN, Knee CR and Joseph AK (2013) Sunscreen use among recreational cyclists: How intentions predict reported behavior. Journal of Health Psychology 18(3): 439-447.

Rhodes RE and Smith NEI (2006) Personality correlates of physical activity: A review and metaanalysis. British Journal of Sports Medicine 40: 958-965.

Sallis J, Prochaska JJ and Taylor WC (2000) A review of correlates of physical activity of children and adolescents. Medicine and Science in Sports and Exercise 32: 963-975.

Smith BN, Kerr NA, Markus MJ, et al. (2001) Individual differences in social loafing: Need for cognition as a motivator in collective performance. Group Dynamics: Theory, Research, \& Practice 5: 150-158.

Stanley C, Pargman D and Tenenbaum G (2007) The effect of attentional coping strategies on perceived exertion in a cycling task. Journal of Applied Sport Psychology 19: 352-363.

Strauss RS, Rodzilsky D, Burack G, et al. (2001) Psychosocial correlates of physical activity in healthy children. Archives of Pediatrics \& Adolescent Medicine 155: 897-902.

Swanson J, Castellanos FX, Murias M, et al. (1998) Cognitive neuroscience of attention deficit hyperactivity disorder and hyperkinetic disorder. Current Opinion in Neurobiology 8: 263-271.

Verplanken B, Hazenberg PT and Palenéwen GR (1992) Need for cognition and external information search effort. Journal of Research in Personality 26: 128-136.

Wang YC, McPherson K, Marsh T, et al. (2011) Health and economic burden of the projected obesity trends in the USA and the UK. Lancet 378: 815-825.

Watt JD and Blanchard MJ (1994) Boredom proneness and the need for cognition. Journal of Research in Personality 28: 44-51.

Weuve J, Kang JH, Manson JE, et al. (2004) Physical activity including walking and cognitive function in older women. The Journal of the American Medical Association 292: 1454-1461. 